\title{
Stereotactic body radiation therapy in head and neck, gynecologic, and pediatric malignancies
}

\author{
Farzan Siddiqui • Charles A. Kunos • Arnold C. Paulino
}

Received: 21 January 2012 / Accepted: 25 January 2012 /Published online: 9 February 2012

(C) Springer-Verlag 2012

\begin{abstract}
Stereotactic body radiotherapy (SBRT) is increasingly being used in the clinic. While most data from this new technology has been in the treatment of early stage lung cancer and sites of oligometastasis in the lung, liver or bone, limited experience exists in other disease sites. Recently, more and more physicians are using SBRT for retreatment. In the head and neck, multiple articles have now been published reporting outcomes in patients with recurrence or those with new primary lesions in previously irradiated areas. The response rates to SBRT have been good; however, long-term control and toxicity are still a major problem. In gynecological malignancies, the literature on SBRT is more limited with initial experience treating para-aortic nodal metastasis from cervical and uterine cancer with excellent local control. Current studies are underway utilizing SBRT with the inclusion of chemotherapy in the treatment regimen of metastatic gynecologic cancers. In pediatrics, where problems of late toxicity are a concern, there is scant information. With all these advances, long-term follow-up is needed to look at complication rates with this form of treatment. In this review, we will discuss the rationale and
\end{abstract}

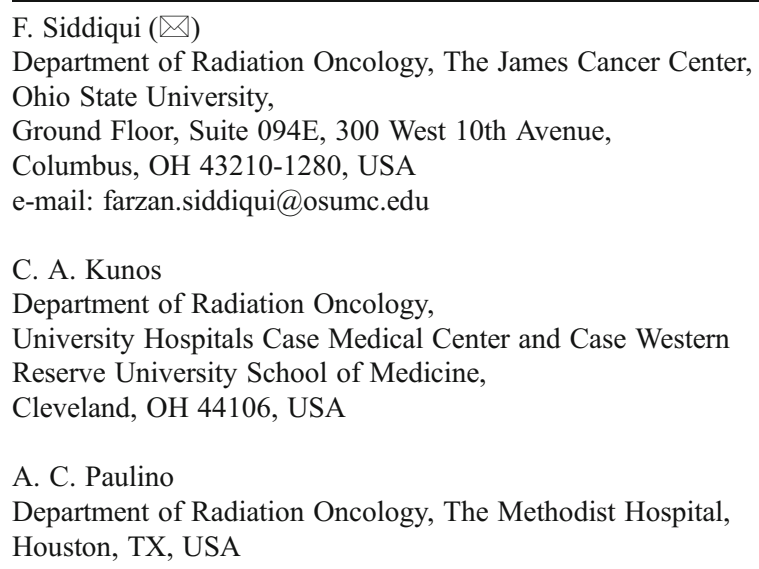

possible applications of SBRT in head and neck, gynecological, and pediatric tumors. We will outline the advantages and disadvantages including possible complications of SBRT. We will also present some illustrative cases on how we have used this technology in our practice.

Keywords Stereotactic · Head and neck cancers · Gynecologic cancers $\cdot$ Pediatric cancers

\section{Introduction}

Stereotactic radiosurgery and stereotactic body radiation therapy (SBRT) have been used in intracranial and base of skull lesions for the past few decades. However, only recently has this technique gained increasing popularity, acceptance, and use in other areas of the body. One of the major reasons for this has been the parallel development of robust image guidance techniques, which allow inter- and intrafraction imaging to ascertain that the target volumes are in the field of radiation. Over the past few years, increasing experience has being obtained in the treatment of head and neck, gynecologic, and pediatric cancers with SBRT. This review discusses some of the recent developments in these areas.

\section{Head and neck cancers}

The annual incidence of head and neck cancers (HNC) of the oral cavity, pharynxes, and larynx in the USA is approximately 52,000, with approximately 11,500 deaths [1]. Globally, these account for approximately 650,000 new cases with 350,000 cancer deaths, representing $6 \%$ of all cancer cases $[1,2]$. About two thirds of these patients 
present with locally advanced stage III or IV disease. Multimodality therapy including surgery, radiation, and chemotherapy form the backbone of treatment for these cancers. However, local recurrence still remains a major pattern of treatment failure in $45 \%$ to $55 \%$ of these patients [3-6]. Treatment options after recurrence are limited. Surgical resection offers the best chance of control. However, this is feasible in only $20 \%$ of cases [7, 8]. Standard fractionated external beam radiation therapy (EBRT) can be delivered with acceptable side effects, but in many cases, the dose delivered is inadequate to control the recurrence. Concurrent EBRT and chemotherapy offer the best chance for control but come at the expense of high risk of side effects and complications. Two RTOG trials (96-10 and 99-11) reported median survivals of 8.2 and 12 months, respectively [9, 10]. Treatment-related mortality was noted in both trials.

Fractionated SBRT is a relatively new approach that is being increasingly explored in patients with recurrent or new primary lesions in previously irradiated areas. Even in newly diagnosed head and neck cancers, there are situations where standard EBRT may not be possible due to medical reasons. In very exceptional and selected cases, SBRT has been used as upfront treatment in these patients [11, 12]. An example of such a situation is where an early stage lesion is well delineated and confined to the site of origin with no lymph node involvement.

Radiobiologically, the use of SBRT in HNC may appear counterintuitive. High doses per fraction are known to result in greater late side effects of treatment. However, spatial restriction and minimal normal tissue irradiation minimizes the risk. Thus, high doses per fraction can be used to cause tumor shrinkage or eradication with acceptable toxicity. This underscores the importance of using all available diagnostic imaging tools including magnetic resonance imaging (MRI), computed tomography (CT) scans, and positron emission tomography (PET)/CT scans to ensure that the tumor is well delineated and its complete extent is ascertained. Use of SBRT in post-operative cases cannot be recommended due to poor and inadequate delineation, post-operative tissue changes, and the fact that normal tissue now occupies the post-operative bed.

Use of stereotactic high dose per fraction radiation therapy continues to increase. Many series have been reported from various part of the world with the largest experiences being from USA and Japan. Previously stereotactic radiosurgery using gamma-knife or linear accelerators was only used in intracranial or base of skull lesions as these sites were amenable to rigid frame-based stereotactic localization. Indeed, large experience exists with the use of SBRT in nasopharyngeal cancers as a boost treatment or for recurrent lesions [13-21]. However, the parallel development and prevalence of new technologies such as kilovoltage and megavoltage cone- beam CT scans, Cyberknife imaging, CT-on-rails, etc. now allows frameless SBRT in extracranial extra-base of skull lesions with improved patient compliance and acceptance. This is reflected in the increasing number of publications in these areas of the head and neck in recent years (Table 1).

\section{Steps involved in SBRT delivery}

1. Patient selection - when considering a patient for HNC SBRT, the following issues need to be addressed.

(a) Newly diagnosed/previously unirradiated-standard treatment options preferred. If these are not feasible due to medical contraindications or poor general condition of patient, SBRT may be considered in exceptional circumstances.

(b) Recurrent or second primary cancers in previously irradiated field - if surgical resection is possible, it should be considered preferentially. If not, the exact extent of the disease must be determined. If the lesion is well-defined and not abutting, critical organs and structures such as the spinal cord/brainstem/carotid artery consider SBRT.

2. Diagnostic imaging - PET/CT scans should be performed to determine the local extent of the metabolically active volume and to rule out any distant metastases. MRI is helpful in defining the soft tissue extent of the disease.

3. Simulation - proper immobilization can be achieved using a thermoplastic mask with custom head-rest. The maxilla and mandible are fixed using a mouth-bite. Further, indentations of the mask into the external auditory canal provide a three-point immobilization of the head and neck. A CT scan is then obtained with iodinated intravenous contrast.

4. Volume definition - the gross tumor volume (GTV) and planning target volume (PTV) are defined after fusing the diagnostic images with the planning CT scan. The total GTV to PTV expansion is usually of the order of 0 to $5 \mathrm{~mm}$ to minimize normal tissue inclusion. Critical normal structures should be carefully delineated to avoid being in the high-dose region or the prescription isodose volume. Linear accelerator-based prescription isodoses range from $80 \%$ to $100 \%$ depending on the institutional experience and clinician preference.

5. Treatment delivery-Four to five fractions of 5 to 8 Gy each are delivered depending on the clinical situation. Treatments are given on alternate days or twice per week separated by two days. Use of image guidance prior to delivery of each fraction is mandatory and can also be considered intrafraction.

6. Response monitoring and follow-up-first follow-up is done at 4-6 weeks post-radiation and MRI and/or $\mathrm{PET} / \mathrm{CT}$ scans are obtained 8 to 12 weeks after treatment. 


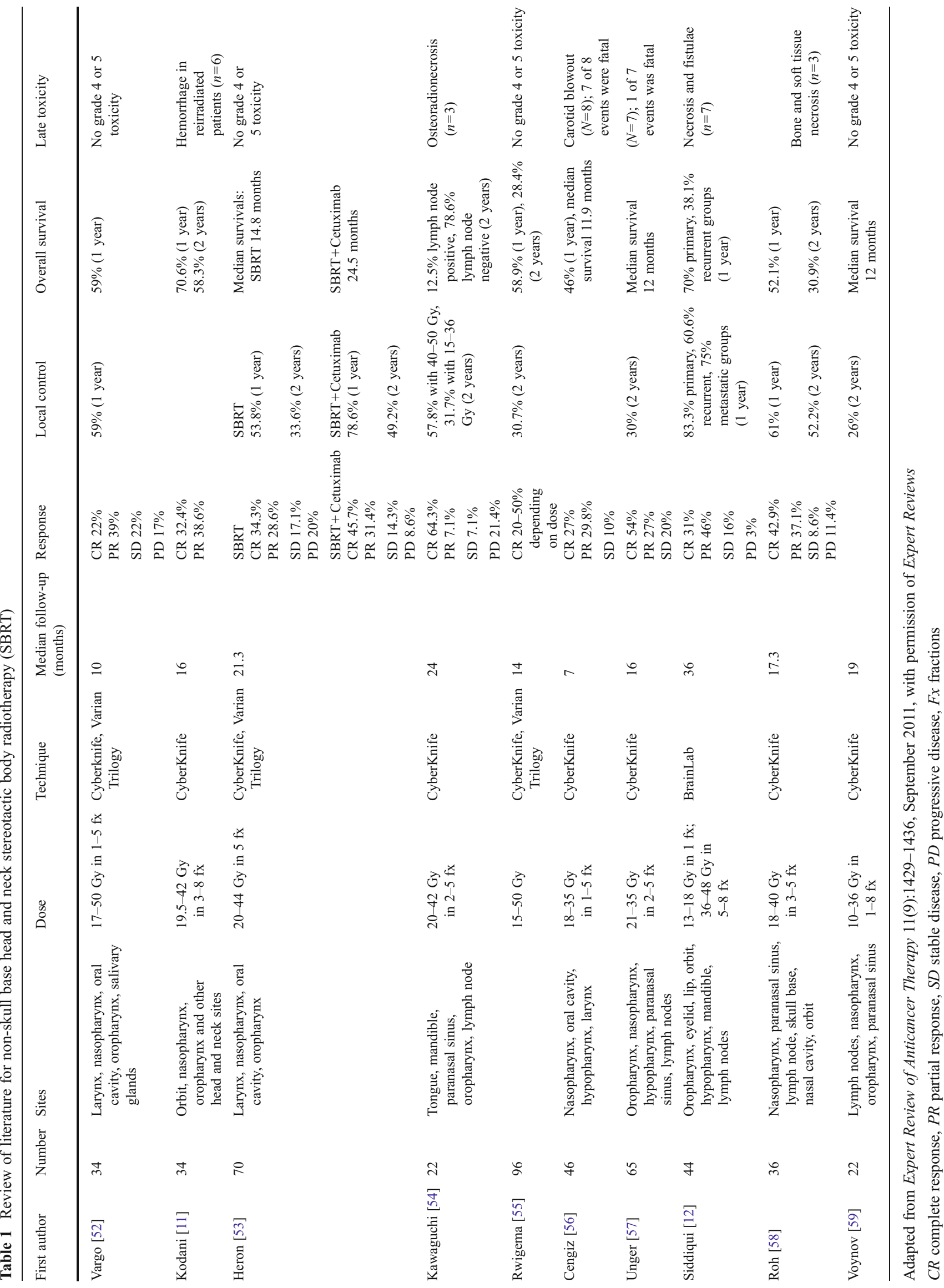




\section{Case illustrations}

Case 1: This case is an example of reirradiation in a previously heavily irradiated field (Fig. 1).

A 69-year-old male was initially diagnosed with nasopharyngeal carcinoma approximately 2.5 years prior to presentation to us. He had received EBRT with concurrent cisplatin chemotherapy to a dose of 70 Gy followed by three adjuvant cycles of cisplatin and 5-fluorouracil. Follow-up 2 years later showed metastatic disease in the lungs, with at least two distinct lesions noted measuring 1.3 and $2 \mathrm{~cm}$ on a PET/CT scan. He was started on chemotherapy using carboplatin and gemcitabine when a repeat PET/CT scan done 6 months later showed progression of both the lung nodules and the presence of a $3-\mathrm{cm}$ left nasopharyngeal mass. MRI done at the same time showed an abnormal soft tissue mass with heterogeneous enhancement with involvement of the left nasopharynx with extension inferolaterally to the left palatine tonsil, left glossotonsillar sulcus, left masticator space, with extension along the margins of the left pharyngeal basilar fascia into the left carotid space.

After explaining all the risks and benefits of fractionated SBRT, the patient was treated using $6 \mathrm{MV}$ photons with an intensity-modulated radiotherapy (IMRT) technique employing eight coplanar fields. The area of recurrence had previously received between 70 to $72 \mathrm{~Gy}$. Carotid sparing IMRT was used to deliver 25 Gy in five fractions prescribed to the $95 \%$ isodose line treating 2 days a week with at least 2 days between each fraction.
Fig. 1 Case details described in the text. a PET/CT scan showing the nasopharyngeal and parapharyngeal recurrence. b Axial and coronal images showing the isodose distribution. c Dose volume histogram. d Eight coplanar IMRT fields used for treatment delivery. e Pre-treatment MRI demonstrating the recurrent nasopharyngeal and parapharyngeal mass. f Follow-up MRI done 4 months post-treatment showing good resolution of the mass with minimal side effects
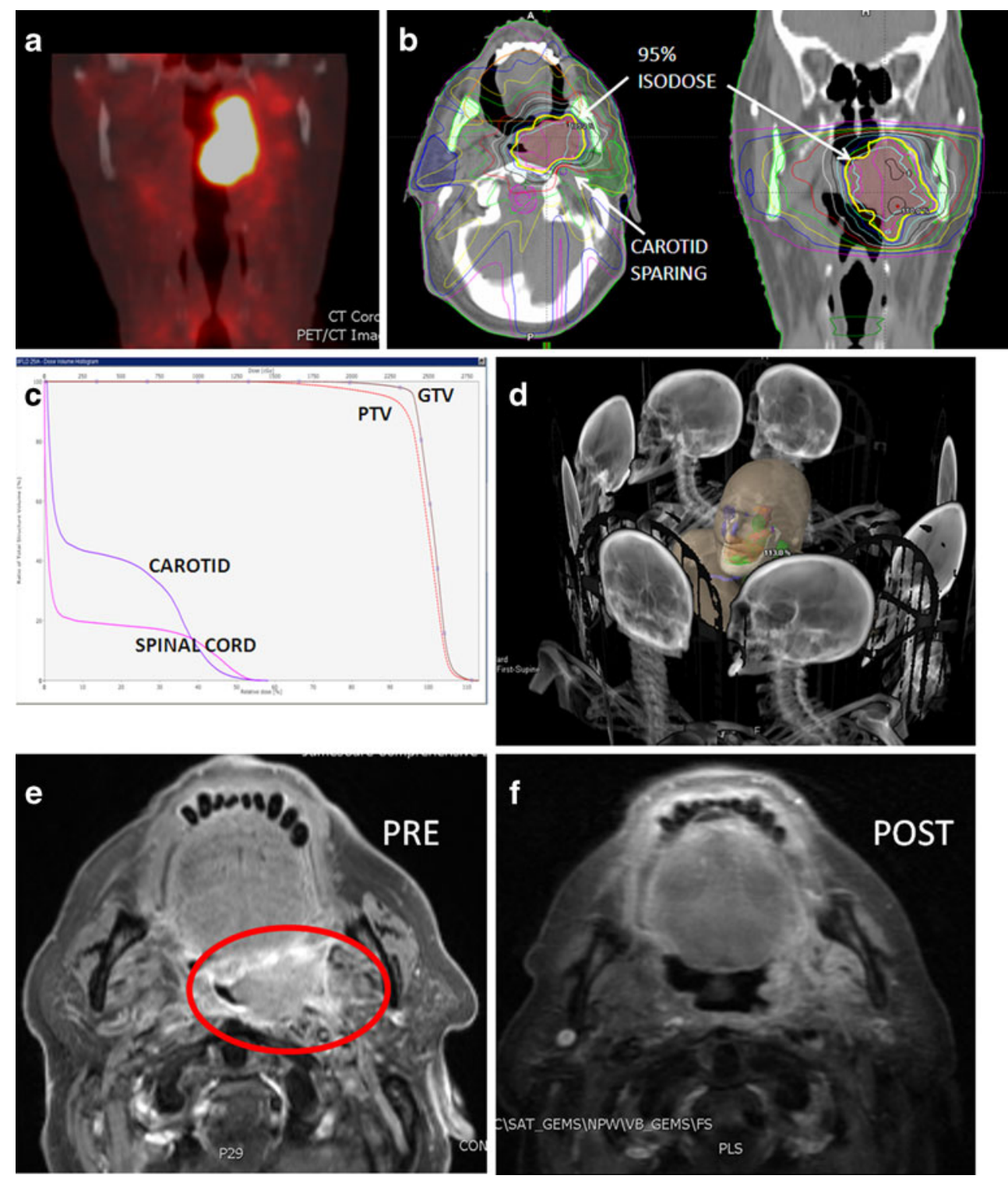
A follow-up MRI done 4 months after treatment showed significant interval reduction in the size of the left nasopharyngeal mass. Chemotherapy was resumed after completion of external beam radiation therapy due to the presence of the multiple lung lesions. On his last follow-up, the patient was doing well with no significant clinical complaints.

Case 2: This case is an example of SBRT as primary treatment for a newly diagnosed head and neck malignancy (Fig. 2).

A 92-year-old male presented with discomfort in the left lower jaw over the past 1 year. He had undergone teeth extraction at this time and ever since the procedure had noted continued pain within the left jaw. Because of the extent of the teeth extraction, pain was attributed to the side effects of his extraction, and he was undergoing symptomatic pain management. When the pain did not significantly improve, the patient had sought another opinion with a neurologist who had performed some initial examinations and placed the patient on Neurontin. This did not do much to alleviate the pain on the left side of his face which continued to increase. Over a period of 1 year, the pain encompassed the entirety of the left face. He then progressed to diplopia which prompted a MRI suggesting an abnormality near the left sinonasal region.

He underwent an endoscopic biopsy of this arising in the pterygopalatine fossa mass which revealed invasive well-differentiated focally keratinizing squamous cell carcinoma. A staging PET/ CT scan done at this time also demonstrated a $2-\mathrm{cm}$ hypermetabolic nodule within the left lung.

On physical examination, cranial nerves III, IV, V-1, V-2, V-3, VI, and VII were affected on the left side. The patient had noticeable ptosis of the left eye and a resting gaze of the left eye in the medial/inferior location, with an inability to significantly move the left eye. There was significant flattening of the left nasolabial fold, although his wrinkling above the eye on the left forehead was still noticeable.

MRI of the brain showed soft tissue signal abnormality that enhanced on post-contrast images seen centered in the region of the left foramen rotundum, which extended to involve the lateral aspect of the left sphenoid sinus, pterygopalatine fossa, left sphenoid bone, infraorbital fissure, left foramen ovale, Meckel's cave, and the vidian canal. There was also involvement of the left cavernous sinus.
The patient was treated using fractionated SBRT. The diagnostic MRI was fused with the planning CT scan and volumes for treatment delineated with help from the neuroradiologist. A total of $30 \mathrm{~Gy}$ was delivered in five fractions of $6 \mathrm{~Gy}$ each prescribed to the $100 \%$ isodose line. As the tumor was encompassing the optic nerve, there was no attempt made to spare the involved optic nerve. Dose to the optic chiasm and contralateral optic nerve were 26.9 and $16.2 \mathrm{~Gy}$, respectively. Nine non-coplanar IMRT fields were used to deliver the treatment.

On follow-up 2 months after completion of treatment, the patient noted improvement in his cranial nerve neuropathies. The left lateral rectus palsy was still present. The patient was able to open his eyes, and the facial droop and nasolabial fold on the left side of the face were much improved. On a follow-up MRI performed approximately 4 months after the radiation treatment, there was redemonstration of soft tissue abnormality. Hence, this patient had clinical and symptomatic improvement in his cranial neuropathy and reduction in facial pain with little demonstrable MRI changes.

This left lung metastasis was also treated using SBRT. On last follow-up, the patient was continuing to work full-time in his car dealership with no significant complaints or side effects of treatment.

\section{Gynecologic cancers}

\section{Rationale for use in gynecologic malignancies}

Radiation treatment often relies on the coupling of external beam radiation and brachytherapy to affect sterilization of pelvic tissue invaded by primary gynecologic cancers (such as cervical, endometrial, ovarian, vaginal, and vulvar cancers). Radiation challenges both cancer cells and normal cells to coordinate a complex cascade of events to repair damaged nuclear and mitochondrial DNA that occurs in forms of altered bases and of single-strand and doublestrand breaks. High-fidelity repair of radiation-induced DNA damage conceptually follows a demand-supply model for economic allocation of intracellular resources, in as much demanded building blocks (i.e., deoxyribonucleotides) either must be newly generated de novo by reduction of ribonucleotides in a reaction catalyzed by ribonucleotide reductase, or must be recovered by salvage kinases linked together in intricate, tightly regulated cytosolic and mitochondrial pathways [22]. For cells, ablative radiosurgery (typically, $>8 \mathrm{~Gy}$ ) ushers in the new repair challenge of cumbersome, possibly overwhelming molecular damage all 
Fig. 2 Case details described in the text. a Axial view of MRI showing diffuse irregular lesion occupying the left base of skull. b Coronal view. c Planning CT scan with axial, coronal, sagittal images and nine-field noncoplanar IMRT fields. d Dose volume histogram. e Follow-up MRI done 4 months post-SBRT did not show appreciable changes. However, patient showed clinical improvement in symptoms
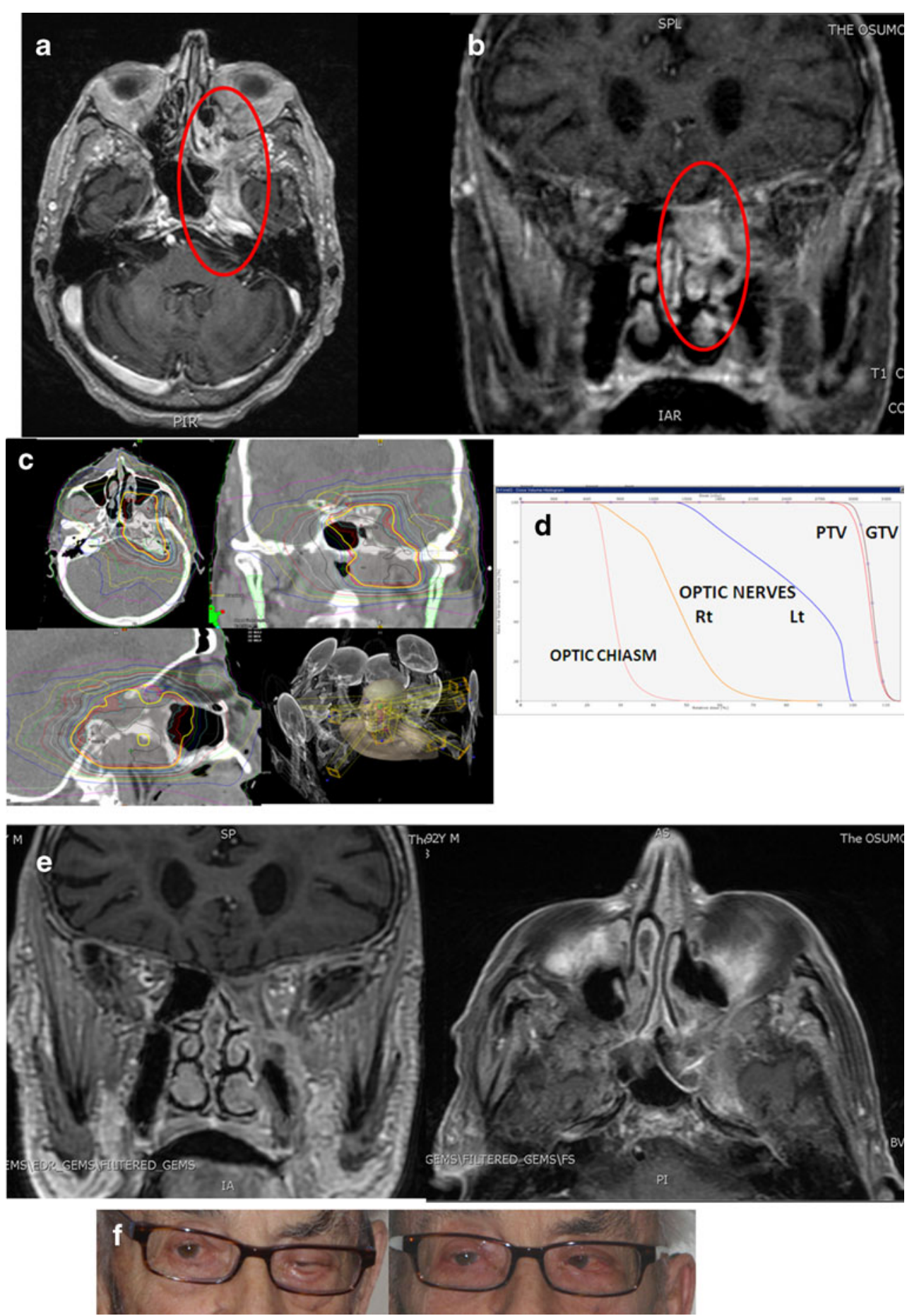

occurring in a narrow time frame. This notion is evocative of short half-times of DNA damage repair being synonymous with intrinsic radiosensitivity [23, 24].

The clinical predicament of high radiation dose delivery to cancer targets without undue pelvic normal tissue injury has been tackled by investigators employing stereotactic body radiosurgery (or stereotactic body radiation therapy). Innovative technology effectively pairing clinical radiation accelerators to helical scanners (Tomotherapy $[25,26]$ ) or to robotic arm mounts (Cyberknife $\left.{ }^{\circledR},[27-30]\right)$ with respiratory gating $[31,32]$ has been a market force for recently-realized radiotherapeutic gains in gynecologic radiation oncology [33]. For purposes of discussion here, the subclass of stereotactic radiosurgery dealing with robotics $\left[\right.$ Cyberknife ${ }^{\circledR}$, Accuray (Sunnyvale, CA, USA)] will be the focus given the preponderance of clinical data accumulated worldwide [27-30]. Robotic cyberknife stereotactic body radiosurgery for gynecologic cancers most commonly involves ablative 
dose (>8 Gy) delivery [34, 35], over a 30-60-min irradiation time span, with sub-millimeter precision $[36,37]$ and with motion tracking [38] all in an effort to avoid injury to normal abdominopelvic tissues. Paramount to the integration of robotic stereotactic body radiosurgery in clinical radiation oncology practice is better understanding of natural systems of DNA damage repair.

Gynecologic cancer cells mend damage done by ionizing radiation in $3 \mathrm{~h}$ through an integrated cascade of proteins, predominantly using ribonucleotide reductase for de novo production of or using thymidine kinase 1 for salvage of deoxyribonucleotides [39-43]. Of the two choices, cells appear to use the de novo ribonucleotide reductase path predominantly [42]. As a putative gatekeeper for deoxyribonucleotide supply, ribonucleotide reductase has a twostep DNA damage response repertoire. First, its M1-M2b complex (alternatively identified, M1-p53R2) aids in repair of DNA damage, whereby DNA damage signals lead to p53 phosphorylation, an alleged release of "bound" M2b from a p53-M2b protein-protein complex, and generation of deoxyribonucleotides [44]. Second, p53-driven transcription raises its M2 small subunit [45], usually a tightly regulated Sphase specific cell cycle protein [46]. These considerations guided some investigators of ablative radiosurgery to institute ablative 8-Gy radiosurgical fractions given for three consecutive fractions (rather than a high radiation dose single fraction) in an effort to overwhelm precisely targeted cancer cells with deoxyribonucleotide demand outpacing deoxyribonucleotide supply. As an example, Fig. 3 plots gynecologic cancer cell survival after 8 Gy irradiation unperturbed, relative to pharmacologic blockade of ribonucleotide reductase, and relative to diminished deoxynucleotide salvage. Cell survival is better when the de novo or salvage paths are not disrupted. These radiobiology studies support the contention that high radiation dose per fraction may initiate mechanisms of cancer cell death before cells can
Fig. 3 Shown is a linear accelerator mounted on a robotic arm manipulator. a Cervical, endometrial, and ovarian cancer cell survivals on a vertical logarithmic scale are plotted after single fraction 800 cGy irradiation, without perturbation of deoxyribonucleotide production (dark blue) or either with blockade of ribonucleotide reductase by 3 -aminopyridine2-carboxaldehyde thiosemicarbazone ( $5 \mu \mathrm{M}$, aqua blue) for de novo block, or with media-free of deoxynucleosides $([\mathrm{dN}]=0$, green) for impaired salvage as before [42]. b Depicted are radiation doses in centigray for individual "pencil" beams delivered during a 35 -min radiosurgery treatment session. Data chronicle dose at the voxel within tumor receiving maximum point dose. "Pulse" trains of peak dose versus negligible or no doses are evident over the entire treatment session
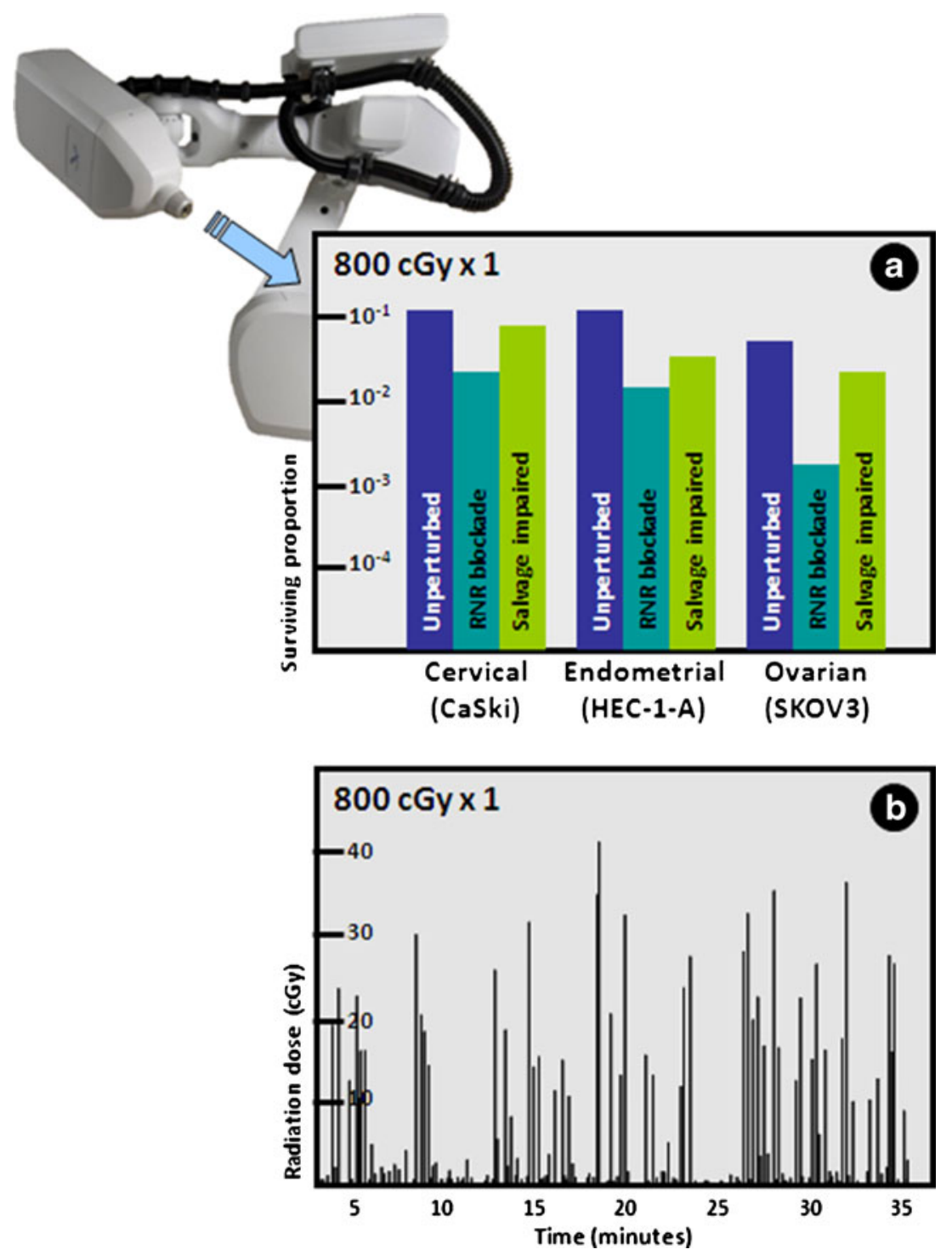
arrest the cell cycle, fix damaged DNA, and then re-initiate the cell cycle. Whether these same effects occur in normal cells is much more difficult to study, but studies are underway. Pilot studies looking into the actual dose to cells delivered over one ablative radiosurgery treatment session reveal "pulse" trains of radiation dose, which by themselves may not be lethal, but their cumulative effect may be so (Fig. 3). Further research is needed to fully characterize these cell repair relationships in regard to ablative radiosurgery.

Given the mechanisms of DNA repair and radiation "pulse" trains, the need to account for the effect of radiosurgical target motion requires consideration of new methods of integrating diagnostic imaging into radiation therapy planning. In devising these new approaches, lessons learnt from the initial study of gynecologic stereotactic radiosurgery should not be ignored [27]. Chief among these lessons is the concept of geographic miss of cancer targets over fixed radiosurgical distances. This is best seen from a body of work done on cutaneous vulvar cancers treated by ablative radiation. Here, sterilizing doses of 8 Gy for three consecutive fractions eradicated visual tumor, but occult disease branching from the primary disease was essentially unaffected and eventually progressed [27]. Expanding clinical tumor volumes, determined by overlays of computed tomography and magnetic resonance imaging or PET/CT, by $3.0 \mathrm{~mm}$ has improved radiosurgical target control [28]. On the device side, real-time cross-plane radiographs for tracking of permanent implanted gold seed fiducials in cancer targets and on-board cameras monitoring quiet breathing affords better targeting of disease. Such principles need to be incorporated in clinical trials of stereotactic body radiosurgery for gynecologic cancer management. Some of these strategies are under development.

\section{Clinical studies of SBRT in gynecologic cancer}

Against this backdrop, clinical gynecologic radiation experience with stereotactic body radiosurgery for disseminated or previously irradiated gynecologic cancers remains limited. One study conducted in Korea [29] evaluated of robotassisted stereotactic body radiosurgery in 30 women having isolated para-aortic lymph node metastases from uterine corpus $(n=2)$ and uterine cervix $(n=28)$ cancers. Typically, extended-field radiation portals substantially reduces hazard for recurrent disease (30\% relapse [47, 48]). In the Korean radiosurgery study, a non-uniform radiosurgical dose of 33 to $45 \mathrm{~Gy}$ in three divided doses provided a $96 \%$ response rate (i.e., greater than $50 \%$ reduction in maximum perpendicular lesion dimension) in targeted para-aortic lymph nodes. Four-year actuarial radiosurgical target control and disease progression-free survival rates were $67 \%$ and $45 \%$, respectively. Median time to progression of disease was
32 months. Adverse events attributable to radiosurgery included five $(17 \%)$ of 30 patients who had grade 3 or higher hematological adverse events. Two other studies, one from the USA [30] and one from Italy (utilizing a conventional linear accelerator [49]), para-aortic lymph node radiosurgical targets were controlled at a rate of $79-84 \%$, with a hazard of non-radiosurgical target progression of $43 \%$ to $63 \%[30,49]$. Toxicities due to radiosurgical treatment were minor and reversible.

Lastly, a prospective phase 2 study of SBRT for treatment of chemorefractory gynecologic malignancies conducted in the USA was presented preliminarily at the 2011 Cyberknife Radiosurgery Summit [35]. An archetypal Cyberknife radiosurgical treatment is illustrated in Fig. 4. As of now, a mixed gynecologic cancer patient population $(n=50)$ received a stereotactic body radiosurgery prescription dose of $24 \mathrm{~Gy}$ ( $8 \mathrm{~Gy} \times 3$ fractions) to para-aortic $(n=21)$, pelvis $(n=14)$, upper abdomen or liver $(n=11)$, and lung $(n=4)$ sites of treatment. No chemotherapy was given during or close to the time of radiosurgery. With a median follow-up of 10 months (range 4 to 24 months), very limited grade toxicities have been encountered. Radiosurgical target treatment responses so far have been clinically meaningful (96\%, 48 of 50 at least partial response by RECIST 1.0 criteria); full durable response assessments are eagerly awaited. And yet, need of new cytotoxic/biologic agent chemotherapy has been needed in $26(52 \%)$ of 50 treated women for non-radiosurgical target metastatic disease progression. The progression of occult metastatic disease in these women has focused attention on co-administration of chemotherapy and radiosurgery. A clinical trial of stereotactic body radiosurgery plus gemcitabine/carboplatin chemotherapy is under clinical development for women with metastatic gynecologic cancers who have undergone chemotherapy for metastatic disease and have limited-volume persistent cancer targets.

As of now, SBRT remains in clinical development. Implementation of radiosurgery into the anticancer armamentarium needs further clinical trial investigation, which will be facilitated by interested national co-investigators. Comprehensive radiobiological studies characterizing DNA damage responses and peculiarities of ablative radiation delivery remain research topics of high medical interest.

\section{Pediatric tumors}

Unique issues of SBRT in children

While most of the experience with SBRT has been in adults with lung, liver, and bone tumors, there is paucity of data in the literature regarding SBRT in children. The subspecialty of 
Fig. 4 Robotic cyberknife radiosurgery for endometrial cancer relapse in a prior fourfield box radiation field is shown. a One hundred noncoplanar beams converge on a single endometrial cancer target located at the proximal vagina. Axial (b), coronal (c), and sagittal (d) projections of the radiosurgical target (shaded in red) and the isodose contour lines of the prescription dose of 24 Gy (thick red contour line). e Dose volume histograms. Contouring for rectum (brown), bladder (orange), small and large bowel (light blue), right (dark blue) and left (magenta) femoral heads, and skin (mustard) are illustrated
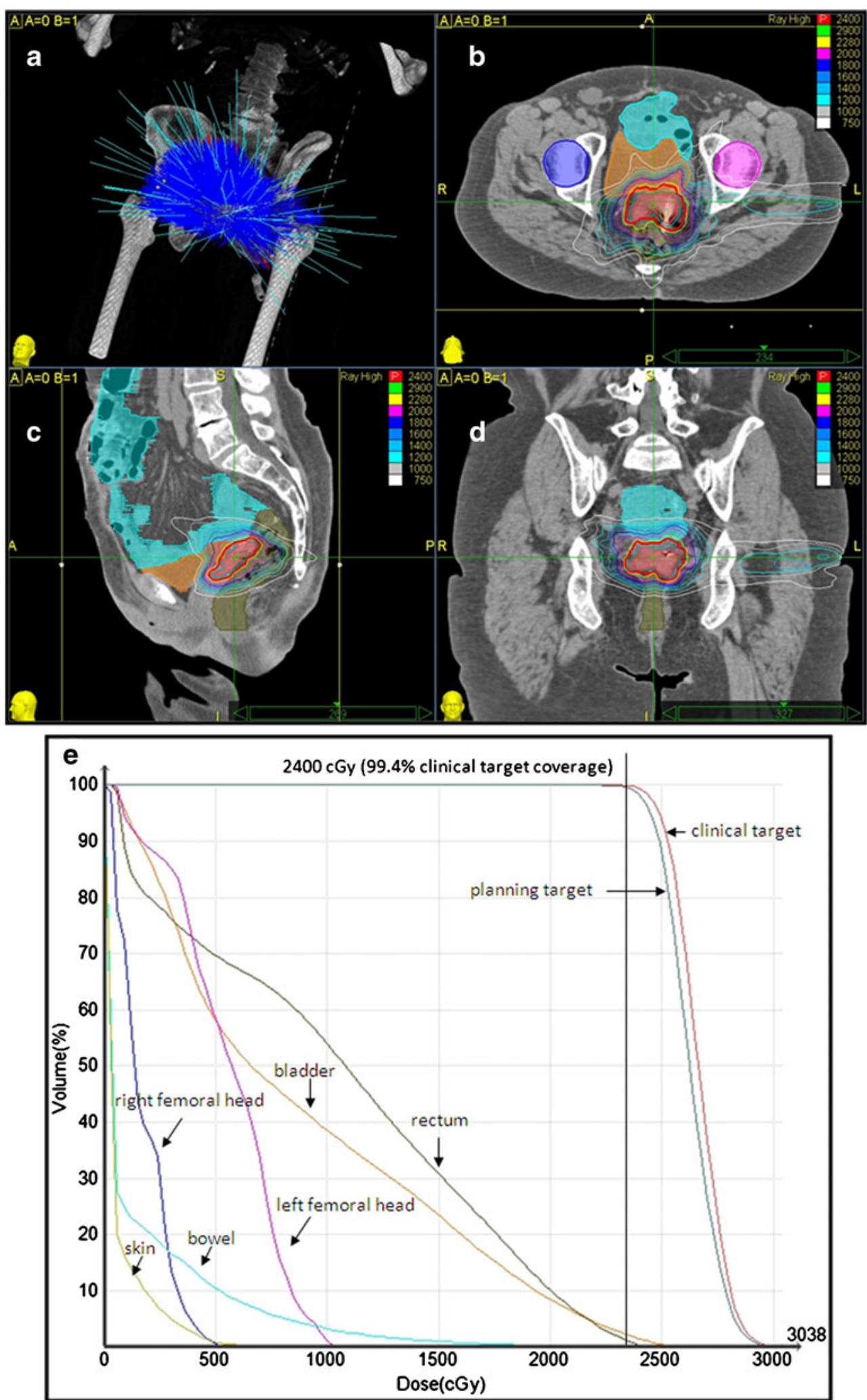

pediatric radiation oncology is unique for multiple reasons. Tumors in children occur in different body sites, and the lessons learned from treating adults using SABR in the same sites may have some applicability. However, there are four important considerations in the treatment of pediatric tumors with SABR.

First, SBRT requires excellent immobilization. One potential problem in children, especially the very young, is inability 
to cooperate and stay still. In such situation, the child will need to be sedated. In our institution, these patients are given general anesthesia using propofol and sevoflurane, both for simulation and treatments.

Second, children and adults get different kinds of tumors. Many of the tumors which occur in children are considered to be more radiosensitive and may need lower doses of radiation. Wilms' tumor and neuroblastoma usually require doses anywhere from 10 to 36 Gy while musculoskeletal tumors such as rhabdomyosarcoma and Ewing sarcoma require 50.4 to 55.8 Gy using conventional fractionation. There are, however, some tumors that have been classically classified as more radioresistant such as osteosarcoma and non-rhabdomyosarcoma soft tissue sarcoma (NRSTS) and may need doses which range from 60 to $70 \mathrm{~Gy}$. In the setting of gross disease from osteosarcoma or NRSTS, SBRT using hypofractionated doses of 10 to 12 Gy per fraction for four or five fractions may have more impact on local control than a 70 Gy in 35 fraction schedule.

Third, while many of the pediatric tumors use lower radiation doses, treatments have been given in the setting of concurrent chemotherapy. In adults treated with SBRT either for primary or oligometastatic disease, radiotherapy is often used alone. It is unclear whether the use of chemotherapy during SBRT in pediatric neoplasms will enhance both acute and late toxicity. On the other hand, if radiotherapy is used alone in the form of SBRT, it is unknown whether the doses for pediatric tumors need to be more similar to adult tumors as chemotherapy might allow for some radiotherapy dose reduction.
Finally, the normal tissues of children are unlike that of adults, and the late effects of hypofractionated radiotherapy need to be taken into consideration. It is well-known that the fraction size is an important determinant of late toxicity. While the late effects in adults tend to be inflammatory and fibrogenic, a unique mechanism of late effects in children is delayed or impaired maturation [50]. For example, while radiation-related osteonecrosis and fracture can occur both in adults and children, bone hypoplasia is seen primarily in individuals whose skeletal tissues are still growing. It is also unknown how SBRT may impact on the risk of carcinogenesis. Children are particularly more susceptible than adults in development of secondary malignancies. This is partly because of genetic susceptibility as many of the pediatric tumors have germline mutations. It is believed that certain radiation-induced cancers may be seen more frequently depending on whether higher volumes of normal tissue are getting low-dose or high-dose radiotherapy. In general, an increase in volumes of tissue receiving low-dose radiation may be associated with a higher risk of developing radiationinduced leukemias and carcinomas, while an increase in volumes of normal tissue receiving high-dose radiation may be associated with a higher risk of radiation-induced sarcomas [51]. While the volume of tissue getting high doses of radiation is definitely smaller in SBRT compared to intensity modulated radiotherapy (IMRT) or three-dimensional, conformal radiotherapy, the biologically equivalent dose is higher because of the increased dose per fraction given in a shorter period of time. Whether this may impact on an
Fig. 5 Stereotactic body radiotherapy $(S B R T)$ in a 16year-old child with Ewing sarcoma and pulmonary relapse previously treated with chemotherapy and whole lung irradiation. After salvage chemotherapy, only one nodule in the left lower lobe of the lung, adjacent to the heart was remaining. The gross tumor volume (GTV; orange), planning target volume (PTV; burnt orange), heart (light green), and lungs (dark green) are outlined. The PTV was determined by adding margin for lung motion through the use of 4-D CT during simulation. A dose of $30 \mathrm{~Gy}$ in five fractions prescribed to the GTV and PTV was delivered; treatments were given Monday, Wednesday, and Friday. a Coronal slice, b sagittal slice, $\mathbf{c}$ axial slice, $\mathbf{d}$ dose volume histogram
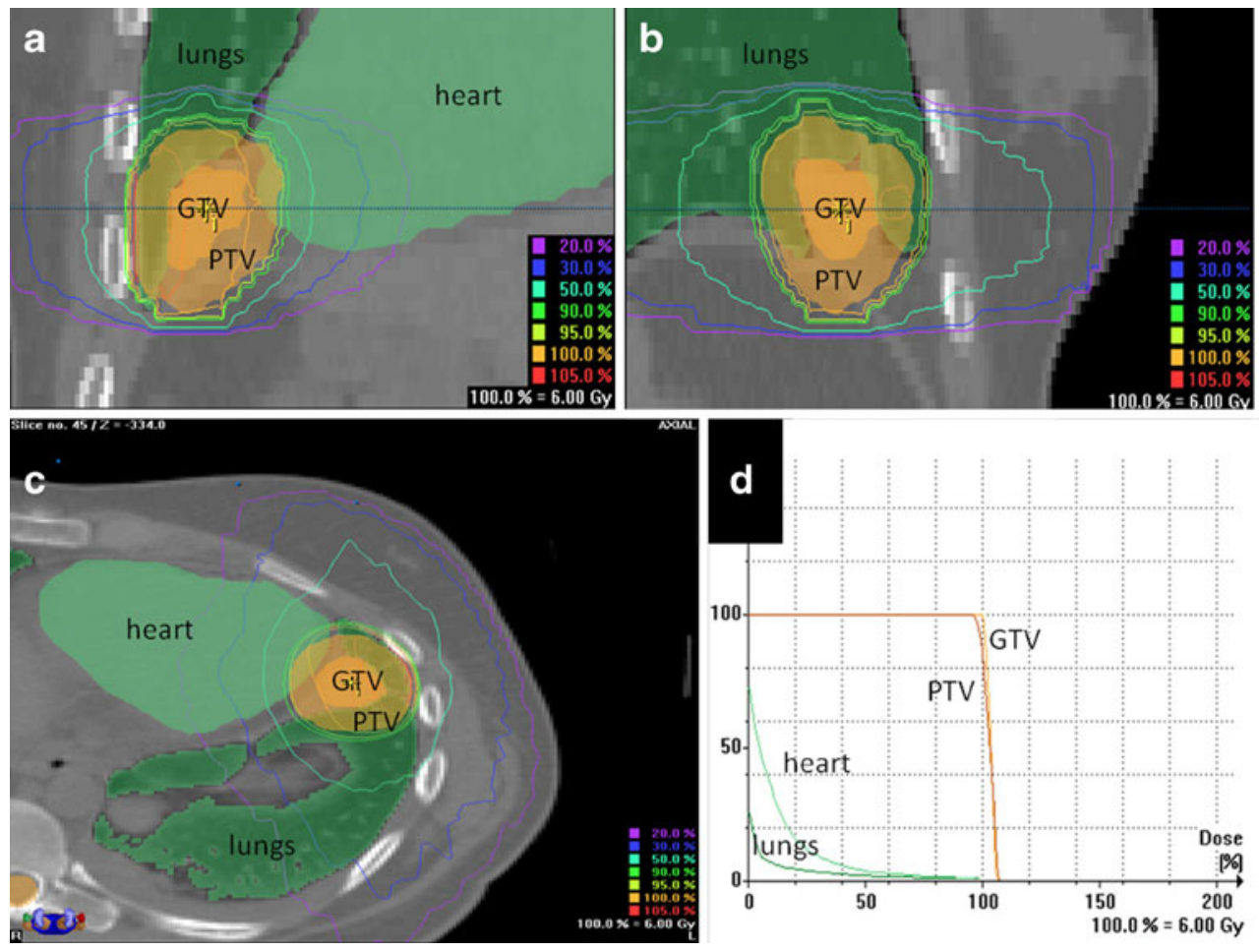
increase in the number of radiation-induced sarcomas is unknown and needs further clarification. In summary, the question of whether the doses for pediatric tumors need to be lowered in SBRT secondary to radiosensitivity in many tumors and concern for late toxicity is an important one and needs to be addressed in future trials. Unfortunately, there are no studies which currently exist on use of SBRT for pediatric malignancies.

Future direction: potential applicability of SBRT in pediatrics

There are two clinical situations where SBRT might be beneficial in the management of childhood cancer. The first situation is in the setting of residual disease after primary or salvage treatment. Although surgery is usually the treatment of choice, radiotherapy can be used for the medically unfit or those with unresectable tumors. An example is a patient with Ewing sarcoma and pulmonary metastases treated with chemotherapy and radiotherapy to the primary site and bilateral lungs. In addition, there is a solitary bone metastasis to the spine where resection is difficult, and furthermore, the risk of complications may not be acceptable given the presence of metastatic disease. SBRT can be used to treat the spinal lesion. Another example is shown in Fig. 5. A 16-year-old patient has relapsed Ewing sarcoma in the lungs and has received previous whole lung irradiation dose of 1,500 cGy in ten fractions. Salvage chemotherapy was given with clearance of all but one of the pulmonary lesions. Surgery was not offered to her because of her history of systemic relapsed disease. PET/CT revealed uptake in the remaining left lower lobe, posterior lung lesion. SBRT was employed delivering 30 Gy in five fractions over 9 days. Repeat PET/CT 2 months later revealed obliteration of the pulmonary nodule.

Another situation where SBRT may have a role is in the treatment of oligometastatic disease. For example, in the treatment of isolated metastatic disease in the liver or lung, metastectomy is often the treatment of choice. However, there may be some situation where metastectomy is not feasible or not preferable because of tumor location such as in the bone (i.e., skull, spine, pelvis) or lung and liver (central disease).

\section{References}

1. Siegel R, Ward E, Brawley O et al (2011) Cancer statistics, 2011: the impact of eliminating socioeconomic and racial disparities on premature cancer deaths. CA Cancer J Clin 61:212-236

2. Parkin DM, Bray F, Ferlay J et al (2005) Global cancer statistics, 2002. CA Cancer J Clin 55:74-108

3. Adelstein DJ, Li Y, Adams GL et al (2003) An intergroup phase III comparison of standard radiation therapy and two schedules of concurrent chemoradiotherapy in patients with unresectable squamous cell head and neck cancer. J Clin Oncol 21:92-98
4. Bonner JA, Harari PM, Giralt J et al (2006) Radiotherapy plus cetuximab for squamous-cell carcinoma of the head and neck. $\mathrm{N}$ Engl J Med 354:567-578

5. Calais G, Alfonsi M, Bardet E et al (1999) Randomized trial of radiation therapy versus concomitant chemotherapy and radiation therapy for advanced-stage oropharynx carcinoma. J Natl Cancer Inst 91:2081-2086

6. Fu KK, Pajak TF, Trotti A et al (2000) A Radiation Therapy Oncology Group (RTOG) phase III randomized study to compare hyperfractionation and two variants of accelerated fractionation to standard fractionation radiotherapy for head and neck squamous cell carcinomas: first report of RTOG 9003. Int J Radiat Oncol Biol Phys 48:7-16

7. Taussky D, Dulguerov P, Allal AS (2005) Salvage surgery after radical accelerated radiotherapy with concomitant boost technique for head and neck carcinomas. Head Neck 27:182-186

8. Temam S, Pape E, Janot F et al (2005) Salvage surgery after failure of very accelerated radiotherapy in advanced head-and-neck squamous cell carcinoma. Int J Radiat Oncol Biol Phys 62:1078-1083

9. Langer CJ, Harris J, Horwitz EM et al (2007) Phase II study of low-dose paclitaxel and cisplatin in combination with split-course concomitant twice-daily reirradiation in recurrent squamous cell carcinoma of the head and neck: results of Radiation Therapy Oncology Group Protocol 9911. J Clin Oncol 25:4800-4805

10. Spencer SA, Harris J, Wheeler RH et al (2001) RTOG 96-10: reirradiation with concurrent hydroxyurea and 5-fluorouracil in patients with squamous cell cancer of the head and neck. Int $\mathrm{J}$ Radiat Oncol Biol Phys 51:1299-1304

11. Kodani N, Yamazaki H, Tsubokura T et al (2009) Stereotactic body radiation therapy for head and neck tumor: disease control and morbidity outcomes. J Radiat Res (Tokyo) 52:24-31

12. Siddiqui F, Patel M, Khan M et al (2009) Stereotactic body radiation therapy for primary, recurrent, and metastatic tumors in the head-and-neck region. Int J Radiat Oncol Biol Phys 74:10471053

13. Buatti JM, Friedman WA, Bova FJ et al (1995) Linac radiosurgery for locally recurrent nasopharyngeal carcinoma: rationale and technique. Head Neck 17:14-19

14. Chua DT, Sham JS, Kwong PW (2003) Linear accelerator-based stereotactic radiosurgery for limited, locally persistent, and recurrent nasopharyngeal carcinoma: efficacy and complications. Int $\mathrm{J}$ Radiat Oncol Biol Phys 56:177-183

15. Hara W, Loo BW Jr, Goffinet DR et al (2008) Excellent local control with stereotactic radiotherapy boost after external beam radiotherapy in patients with nasopharyngeal carcinoma. Int J Radiat Oncol Biol Phys 71:393-400

16. Kondziolka D, Lunsford LD (1991) Stereotactic radiosurgery for squamous cell carcinoma of the nasopharynx. Laryngoscope 101:519-522

17. Le QT, Tate D, Koong A et al (2003) Improved local control with stereotactic radiosurgical boost in patients with nasopharyngeal carcinoma. Int J Radiat Oncol Biol Phys 56:1046-1054

18. Pai PC, Chuang CC, Wei KC et al (2002) Stereotactic radiosurgery for locally recurrent nasopharyngeal carcinoma. Head Neck 24:748-753

19. Tate DJ, Adler JR Jr, Chang SD et al (1999) Stereotactic radiosurgical boost following radiotherapy in primary nasopharyngeal carcinoma: impact on local control. Int J Radiat Oncol Biol Phys 45:915-921

20. Wu SX, Chua DT, Deng ML et al (2007) Outcome of fractionated stereotactic radiotherapy for 90 patients with locally persistent and recurrent nasopharyngeal carcinoma. Int J Radiat Oncol Biol Phys 69:761-769

21. Yau TK, Sze WM, Lee WM et al (2004) Effectiveness of brachytherapy and fractionated stereotactic radiotherapy boost for persistent nasopharyngeal carcinoma. Head Neck 26:1024-1030 
22. Kunos C, Radivoyevitch T (2011) Molecular strategies of deoxynucleotide triphosphate supply inhibition used in the treatment of gynecologic malignancies. Gynecol Obstet (in press)

23. Fowler J (1993) Why shorter half-times of repair lead to greater damage in pulsed brachytherapy. Int $\mathrm{J}$ Radiat Oncol Biol Phys 26:353-356

24. Fowler J, Welsh J, Howard S (2004) Loss of biological effect in prolonged fraction delivery. Int J Radiat Oncol Biol Phys 59:242249

25. Kim Y, Kim J, Jeong K et al (2009) Dosimetric comparisons of three-dimensional conformal radiotherapy, intensity-modulated radiotherapy, and helical tomotherapy in whole abdominopelvic radiotherapy for gynecologic malignancy. Technol Cancer Res Treat 8:369-377

26. Hsieh C, Wei M, Hsu Y et al (2010) Should helical tomotherapy replace brachytherapy for cervical cancer? Case report. BMC Cancer 10:637

27. Kunos C, Von Gruenigen V, Waggoner S et al (2008) Cyberknife radiosurgery for squamous cell carcinoma of the vulva after prior pelvic radiation therapy. Technol Cancer Res Treat 7:375-380

28. Kunos C, Chen W, DeBernardo R et al (2009) Stereotactic body radiosurgery for pelvic relapse of gynecologic malignancies. Technol Cancer Res Treat 8:393-400

29. Choi C, Cho C, Yoo S et al (2009) Image-guided stereotactic body radiation therapy in patients with isolated para-aortic lymph node metastases from uterine cervical and corpus cancer. Int J Radiat Oncol Biol Phys 74:147-153

30. Higginson D, Morris D, Jones E et al (2011) Stereotactic body radiotherapy (SBRT): Technological innovation and application in gynecologic oncology. Gynecol Oncol 120:404-412

31. Wunderink W, Mendez Romero A, de Kruijf W et al (2008) Reduction of respiratory liver tumor motion by abdominal compression in stereotactic body frame, analyzed by tracking fiducial markers implanted in liver. Int J Radiat Oncol Biol Phys 71:907-915

32. Ozhasoglu C, Saw C, Hungcheng C et al (2008) SynchronyCyberknife respiratory compensation technology. Med Dosimetry 33:117-123

33. Mayr N, Huang Z, Sohn J et al (2011) Emerging application of stereotactic body radiation therapy for gynecologic malignancies. Expert Rev Anticancer Ther 11:1071-1077

34. Kunos C, Zhang Y, Brindle J (2011) Stereotactic body radiosurgery in gynecologic carcinomas. In: Ayhan A, Reed N, Gultekin M, Dursun P (eds) Textbook of gynaecological oncology. Günes, Ankara, pp 835-841

35. Kunos C, Brindle J, Zhang Y et al (2011) A prospective phase 2 evaluation of stereotactic body radiosurgery for gynecologic malignancies. Cyberknife Robotic Radiosurgery Summit

36. Wilcox E, Daskalov G (2007) Evaluation of GAFCHROMIC EBT film for Cyberknife ${ }^{\circledR}$ dosimetry. Med Phys 34:1967-1974

37. Ponsky L, Crownover R, Rosen M et al (2003) Initial evaluation of Cyberknife technology for extracorporeal renal tissue ablation. Urology 61:498-501

38. Hoogeman M, Prévost J-B, Nuyttens J et al (2009) Clinical accuracy of the respiratory tumor tracking system of the Cyberknife: assessment by analysis of log files. Int J Radiat Oncol Biol Phys 74:297-303

39. Kunos C, Chiu S, Pink J et al (2009) Modulating radiation resistance by inhibiting ribonucleotide reductase in cancers with virally or mutationally silenced p53 protein. Radiation Res 172:666-676

40. Kunos C, Radivoyevitch T, Pink J et al (2010) Ribonucleotide reductase inhibition enhances chemoradiosensitivity of human cervical cancers. Radiation Res 174:574-581

41. Kunos C, Colussi V, Pink J et al (2011) Radiosensitization of human cervical cancer cells by inhibiting ribonucleotide reductase: enhanced radiation response at low dose rates. Int J Radiat Oncol Biol Phys 80:1198-1204

42. Kunos C, Ferris G, Pyatka N et al (2011) Deoxynucleoside salvage facilitates DNA repair during ribonucleotide reductase blockade in human cervical cancers. Radiat Res 176:425-433

43. Boothman D, Davis T, Sahijdak W (1994) Enhanced expression of thymidine kinase in human cells following ionizing radiation. Int $\mathrm{J}$ Radiat Oncol Biol Phys 30:391-398

44. Xue L, Zhou B, Liu X et al (2003) Wild-type p53 regulates human ribonucleotide reductase by protein-protein interaction with $\mathrm{p} 53 \mathrm{R} 2$ as well as hRRM2 subunits. Cancer Res 63:980-986

45. Kuo M-L, Kinsella T (1998) Expression of ribonucleotide reductase after ionizing radiation in human cervical carcinoma cells. Cancer Res 58:2245-2252

46. Chabes A, Thelander L (2000) Controlled protein degradation regulates ribonucleotide reductase activity in proliferating mammalian cells during the normal cell cycle and in response to DNA damage and replication blocks. J Biol Chem 275:17747-17753

47. Grigsby PW, Heydon K, Mutch DG et al (2001) Long-term followup of RTOG 92-10: cervical cancer with positive para-aortic lymph nodes. Int J Radiat Oncol Biol Phys 51:982-987

48. Varia MA, Bundy BN, Deppe G (1998) Cervical carcinoma metastatic to para-aortic nodes: extended field radiation therapy with concomitant 5-fluorouracil and cisplatin chemotherapy: a Gynecologic Oncology Group study. Int J Radiat Oncol Biol Phys 42:1015-1023

49. Deodato F, Macchia G, Grimaldi L et al (2009) Stereotactic radiotherapy in recurrent gynecological cancer: a case series. Oncol Rep 22:415-419

50. Paulino AC, Constine LS, Rubin P et al (2010) Normal tissue development, homeostasis, senescence, and the sensitivity to radiation injury across the age spectrum. Semin Radiat Oncol 20:1220

51. Hall EJ, Wuu CS (2003) Radiation-induced second cancers: the impact of 3D-CRT and IMRT. Int J Radiat Oncol Biol Phys 56:8388

52. Vargo JA, Wegner RE, Heron DE et al (2011) Stereotactic body radiation therapy for locally recurrent, previously irradiated nonsquamous cell cancers of the head and neck. Head Neck. doi:10.1002/hed.21889

53. Heron DE, Rwigema JC, Gibson MK et al (2011) Concurrent cetuximab with stereotactic body radiotherapy for recurrent squamous cell carcinoma of the head and neck: a single institution matched case-control study. Am J Clin Oncol 34:165-172

54. Kawaguchi K, Sato K, Horie A et al (2010) Stereotactic radiosurgery may contribute to overall survival for patients with recurrent head and neck carcinoma. Radiat Oncol 5:51

55. Rwigema JC, Heron DE, Ferris RL et al (2011) The impact of tumor volume and radiotherapy dose on outcome in previously irradiated recurrent squamous cell carcinoma of the head and neck treated with stereotactic body radiation therapy. Am J Clin Oncol 34:372-379

56. Cengiz M, Ozyigit G, Yazici G et al (2011) Salvage reirradiaton with stereotactic body radiotherapy for locally recurrent head-andneck tumors. Int J Radiat Oncol Biol Phys 81:104-109

57. Unger KR, Lominska CE, Deeken JF et al (2010) Fractionated stereotactic radiosurgery for reirradiation of head-and-neck cancer. Int J Radiat Oncol Biol Phys 77:1411-1419

58. Roh KW, Jang JS, Kim MS et al (2009) Fractionated stereotactic radiotherapy as reirradiation for locally recurrent head and neck cancer. Int J Radiat Oncol Biol Phys 74:1348-1355

59. Voynov G, Heron DE, Burton S et al (2006) Frameless stereotactic radiosurgery for recurrent head and neck carcinoma. Technol Cancer Res Treat 5:529-535 